\title{
Validity of carbohydrate deficient transferrin and other markers as diagnostic aids in the detection of alcohol related seizures
}

\author{
Geir Bråthen, Kristian S Bjerve, Eylert Brodtkorb, Gunnar Bovim
}

\begin{abstract}
Objective-The role of alcohol misuse in the genesis of seizures is probably often undetected. The aim was to investigate the utility of carbohydrate deficient transferrin (CDT) compared with other biomarkers and clinical examination in the diagnosis of alcohol related seizures.

Methods-The study included consecutively 158 seizure patients -83 men and 75 women-with mean age 45 (16-79) years. Seizures related to alcohol use were identified by a score $\geqslant 8$ in the alcohol use disorders identification test (AUDIT positive). AUDIT was applied as the gold standard to which sensitivity and specificity of the various markers were related. Blood samples were obtained from 150 patients on admission and analysed for ethanol, liver enzymes, and CDT, using AXIS Biochemicals' \%CDT-TIA kit.

Results-53 patients (34\%) were AUDIT positive. Using the commonly applied decision value for \%CDT of $5.0 \%$, a sensitivity of $41 \%$ and a specificity of $84 \%$ were obtained. Analysis of receiver operator characteristics (ROC) curves disclosed an optimal cut off value for \%CDT of $5.4 \%$, which yielded a sensitivity of $39 \%$ and a specificity of $88 \%$. At a specificity of $80 \%$, the sensitivity was $43 \%$ for $\%$ CDT and $26 \%$ for GGT. The \%CDT sensitivity was markedly higher for men than for women. Compared with GGT, ASAT, ALAT, and ASAT/ALAT ratio, CDT was the best single biomarker for alcohol related seizures. However, even in the subgroup of withdrawal seizures, the sensitivity level barely exceeded $50 \%$. Clinicians scored alcohol as the main cause of the seizure in only 19 cases $(12 \%)$. In $38(24 \%)$ cases, clinicians suspected that alcohol had a role (sensitivity of $62 \%$ at a specificity of $89 \%$ ). Their ability to identify AUDIT positive patients was better than that of any biomarker, but many cases were missed. Agreement of clinicians' scores to CDT was only fair $(\kappa=0.28)$. CDT concentrations were significantly increased among alcohol abstaining patients on enzymeinducing antiepileptic drugs. Six out of 16 patients with false positive CDT results were exposed to such drugs.
\end{abstract}

Conclusions-CDT is not recommended as a stand alone marker for alcohol related seizures, but may provide a useful contribution to the overall diagnostic investiga- tion of seizures. Confirmatory studies are needed as to the apparent vulnerability of CDT to antiepileptic drugs.

(F Neurol Neurosurg Psychiatry 2000;68:342-348)

Keywords: carbohydrate deficient transferrin; alcohol; seizures; biochemical markers

The association of seizures with alcohol misuse is well established. ${ }^{12}$ A high proportion of seizures leading to hospital admission may be related to alcohol use..$^{3-5}$ Alcohol misuse as seizure aetiology is often undetected and has been associated with a higher crude mortality than seizures of unknown aetiology. ${ }^{6}$ Prophylactic treatment of seizures provoked by alcohol use should be limited to a few days when needed. ${ }^{7}$ Due to non-compliance, undue antiepileptic drug (AED) treatment may even contribute to seizure provocation. Adequate and early counselling is essential in the management of these patients. Therefore, identification of alcohol related seizures is important and, as the clinical diagnosis is difficult, improved diagnostic tools are needed.

Carbohydrate deficient transferrin (CDT) has been proposed as the most specific biomarker of sustained alcohol misuse. ${ }^{8}$ In well contrasted populations of middle aged male alcohol misusers versus control subjects, sensitivity and specificity have been reported as high as $>80 \%$ and $>90 \%$, respectively. ${ }^{9}$ However, in identifying hazardous or harmful drinking in general medical settings, CDT seems to be of more limited value. ${ }^{9-13}$

Most alcohol related seizures have been reported to occur within a time frame of 48 hours after cessation of drinking. ${ }^{14}$ Epidemiological studies indicate that the relation between seizures and alcohol use may be dose dependent. ${ }^{15}$ Based on these findings, this patient group should be ideal for studies of CDT as a diagnostic tool. Our aims were to study the validity of CDT in detecting alcohol related seizures, in comparison with other markers, and to the skills of the experienced clinician using clinical examination and routine analyses.

\section{Material and methods}

SUBJECTS

During the study period from January 1995 to October 1996, 225 consecutive patients who were admitted to hospital with seizures were recruited. Twenty eight patients were disabled due to encephalopathy, psychosis, dementia, or severe somatic disease. Participation was 
voluntary, and informed consent was obtained. Out of the 197 patients who were invited to participate in the study, 37 refused, and in two patients, important data were missing after inclusion. Thus, 158 patients -83 men and 75 women-were included. Any patient admitted for seizures was eligible for the study, including 16 patients later shown to have other diagnoses (eight convulsive syncopes and eight psychogenic seizures). Licence to collect and store patient data was granted by The Norwegian Data Inspectorate. The regional committee for ethics in medical research approved the study.

THE INTERVIEW

The alcohol use disorders identification test (AUDIT) was recorded as part of a detailed semistructured clinical interview, which was performed as soon as possible after recovery from postictal symptoms. Within 3 days after admission, 147 patients (93\%) had been interviewed. AUDIT was developed from a six country World Health Organisation (WHO) collaborative project as a screening instrument for hazardous alcohol consumption. ${ }^{16}$ The 10 item core questionnaire is easily administered and has the advantage of early detection of hazardous drinking, compared with other questionnaire based systems that are mainly developed for detection of alcoholism. We identified subjects with seizures related to alcohol use by AUDIT scores $\geqslant 8$ (AUDIT positive), a cut off by which both sensitivity and specificity for hazardous drinking have been reported as greater than $90 \% .{ }^{17}{ }^{18}$ The amount of alcohol consumed on each of 8 days before admission was carefully registered using standard alcohol units. One unit is equivalent to one small bottle of beer or one glass of wine or spirits equal to $12-15 \mathrm{~g}$ pure ethanol. ${ }^{19}$ The number of hours since the last alcohol intake was recorded. Discrepancies between answers were discussed with the patient to achieve accuracy.

An experienced neurologist scored to what degree the seizure was considered to be related to alcohol use on an ordinal scale from 0 (no relation to alcohol) to 10 (alcohol was the only known cause). In most cases, this neurologist personally examined the patient before the assessment, which was further based on hospital records and routine investigations, including EEG and laboratory analyses. The neurologist had no access to interview data, AUDIT scores, or CDT results. Calculations of validity were made relative to clinician's scores $>0$ (a relation to alcohol use was presumed) and $>5$ (alcohol was considered the most important

Table 1 Characteristics of the study group

\begin{tabular}{llll}
\hline & $\begin{array}{c}\text { AUDIT positive } \\
(n=53)\end{array}$ & $\begin{array}{l}\text { AUDIT negative } \\
(n=105)\end{array}$ & $\begin{array}{l}\text { Difference } \\
(\text { p value })\end{array}$ \\
\hline Men (\%) & 66 & 46 & 0.025 \\
Self reported alcohol misuse (\%) & 57 & 1 & $<0.00005$ \\
History of illegal drug misuse (\%) & 28 & 3 & $<0.0005$ \\
Age (mean (SD)) & $39.8(13.5)$ & $47.6(17.6)$ & 0.002 \\
Intake last week (mean (SD)) units* & $37.4(75.1)$ & $1.9(4.5)$ & $<0.0005$ \\
Cigarettes daily (mean (SD)) & $13.2(10.4)$ & $4.7(7.2)$ & $<0.0005$ \\
\hline
\end{tabular}

*Standard alcohol units. seizure provoking factor). Characteristics of the study group are summarised in table 1 .

DEFINITIONS AND CLASSIFICATION

An operational definition of the term "withdrawal seizures" was applied to seizures occurring in AUDIT positive patients less than 72 hours after the last drink, thus ensuring both a hazardous drinking level and recent intake. Patients reporting no alcohol intake during the preceding year were defined as total abstainers. We studied the effect of AED use on \%CDT concentrations by subdividing patients into three groups; those taking enzyme inducing AEDs, those taking AEDs not considered to be enzyme inducing, and a non-drug group. Carbamazepine, phenytoin, and phenobarbital were considered enzyme inducing, whereas valproate, lamotrigine, vigabatrin, and oxcarbazepine were considered to cause no or little (oxcarbazepine) liver enzyme induction. ${ }^{20}$

BLOOD SAMPLING AND ANALYSIS

Blood samples were collected on admission. Without delay, serum was analysed for $\gamma$-glutamyl transferase (GGT), aspartate aminotransferase (ASAT), alanine aminotransferase (ALAT), and ethanol. Laboratory reference values were applied as cut off concentrations. The reference values were: GGT $<50 \mathrm{U} / 1$ (women) and $<80 \mathrm{U} / 1$ (men), ASAT and ALAT <35 U/1 (women) and <50 U/1 (men). Serum for the CDT analyses was stored at $-80^{\circ} \mathrm{C}$ until analysis. Analyses were performed in our laboratory using the \%CDT-TIA kit produced by AXIS Biochemicals ASA, Oslo, Norway, in accordance with the instructions from the manufacturer. According to the manufacturer, amounts exceeding $6 \%$ are considered increased, whereas abstaining people have values below $5 \%$. We applied $5.0 \%$ as the decision value for hazardous alcohol consumption. However, for comparison some results are also presented using $6.0 \%$ as cut off.

STATISTICAL ANALYSES

Receiver operator characteristic (ROC) curves with calculations and comparisons of areas under ROC curves (AUCs) were produced in the software Medcalc ${ }^{\mathrm{TM}}$ for Windows 95 . The Mann-Whitney test and the Kruskal-Wallis test were applied in non-parametric analyses. Comparisons of amounts of alcohol and biomarkers were done non-parametrically. Correlation coefficients are expressed by Spearman's $r\left(r_{s}\right)$. Agreement rates were calculated from $2 \times 2$ contingency tables and expressed as $\kappa$ values. The two sided significance limit was $\mathrm{p}=0.05$, and confidence intervals (CIs) were $95 \%$.

\section{Results}

CDT COMPARED WITH OTHER BIOMARKERS OF ALCOHOL USE

Fifty three patients were AUDIT positive, whereas 105 were AUDIT negative. \%CDT was the best of the biochemical markers included in this study (table 2). ROC curve analysis showed an optimal cut off point for $\%$ CDT of $5.4 \%$, which yielded a sensitivity of 
Table 2 Validity of biomarkers when laboratory reference values were used as decision limits. Comparison with clinicians'scores $(n=158)$

\begin{tabular}{lllll}
\hline & Decision value & Sensitivity & Specificity & LR \\
\hline (\%) CDT & 5.0 & 0.41 & 0.84 & 2.54 \\
(\%) CDT & 6.0 & 0.33 & 0.91 & 3.67 \\
GT & $\mathrm{f} \leqslant 50, \mathrm{~m} \leqslant 80$ & 0.30 & 0.74 & 1.15 \\
ASAT & $\mathrm{f} \leqslant 35, \mathrm{~m} \leqslant 50$ & 0.28 & 0.92 & 3.50 \\
ALAT & $\mathrm{f} \leqslant 35, \mathrm{~m} \leqslant 50$ & 0.25 & 0.72 & 0.87 \\
ASAT/ALAT ratio & $>1.0$ & 0.50 & 0.50 & 1.00 \\
Ethanol & $>0$ & 0.16 & 0.94 & 2.67 \\
Clinicians' scores & $>0$ & 0.62 & 0.89 & 5.64 \\
Clinicians' scores & $\geqslant 5$ & 0.42 & 1.00 & 3.82 \\
\hline
\end{tabular}

${ }^{\star}$ Clinical assessment of alcohol as cause of seizure on ordinal scale from $0-10$

$\mathrm{LR}=$ likelihood ratio.

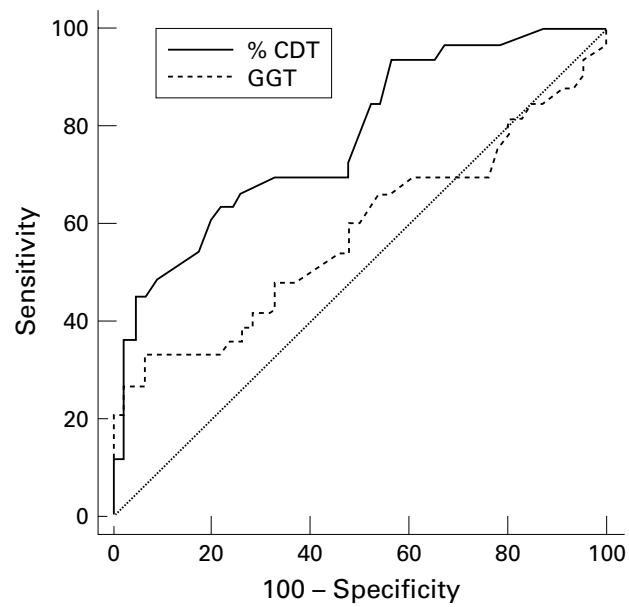

at a specificity of $88 \%$ (likelihood ratio (LR) 3.25). Sensitivity of the various markers were further calculated from ROC curve analyses at specificities of $80 \%$ and $90 \%$, respectively, and are listed in table 3 . Sensitivity of CDT was superior to that of the other biochemical markers. On ROC curve analysis, there was a trend towards difference in AUCs between \%CDT (0.679) and GGT (0.567) in the total group, although not significant $(p=0.063)$. A markedly better validity of both CDT and GT was seen in men (fig 1) than in women (fig 2). In men, AUC for CDT was significantly larger than for GGT $(p=0.011$, see table 3 for values and confidence intervals), whereas in women the ROC curves of CDT and GT were not different $(p=0.903)$.

CDT AND GGT COMPARED WITH EVALUATION BY CLINICIANS

Clinicians scored 19 out of 45 AUDIT positive and none out of 88 AUDIT negative patients as having a $50 \%$ or higher probability of an alcohol related seizure (sensitivity $42 \%$ and specificity $100 \%)$. Twenty eight AUDIT positive and 10 AUDIT negative seizures were considered related to alcohol use to some extent (sensitivity $62 \%$ and specificity $89 \%$ ). Clinicians and biomarkers seemed to identify somewhat different patients, as the agreement of clinicians with $\%$ CDT was fair $(\kappa=0.28)$, whereas the agreement of clinicians with GGT was poor $(\kappa=0.12)$. A subgroup defined by either increased \%CDT, or GGT, or both, correlated
Figure 1 Comparison of ROC curves for $\% C D T$ and $G T$ in men, $n=79$. Difference between areas under curves (AUC (95\%CI);CDT 0.78 (0.67-0.83), GT 0.58 $(0.46-0.69))$ is statistically significant $(p=0.011)$.

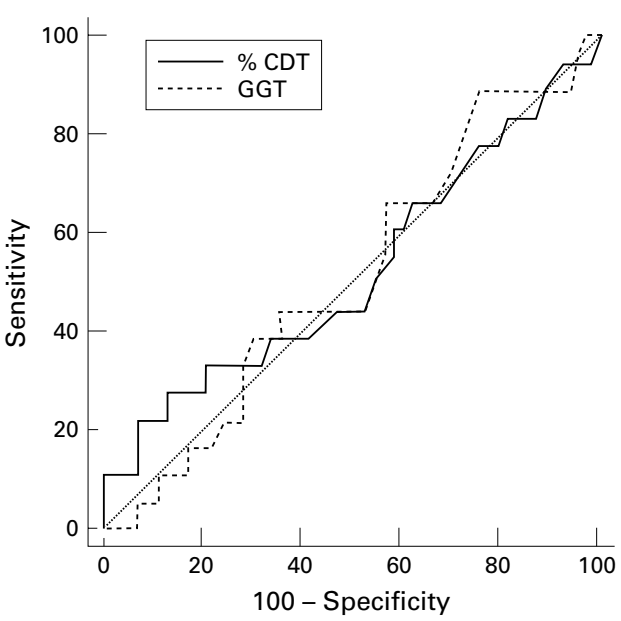

Figure 2 Comparison of ROC curves for \%CDT and GT in women, $n=71$. Areas under curves (AUC (95\%CI); CDT 0.52 (0.40-0.64), GT 0.51 (0.39-0.63) are not different $(p=0.90)$. Both for CDT and for GT, markedly lower areas under curves are apparent for women than for men, but the sex difference is significant only for $\% C D T$.

better with AUDIT $\left(r_{\mathrm{s}}=0.33, \mathrm{p}<0.001\right)$ than did each of the two variables $\%$ CDT $\left(r_{s}=0.27\right.$,

Table 3 Sensitivity of biomarkers relative to AUDIT at $80 \%$ and $90 \%$ specificity. Data derived from ROC analyses. Clinician's ability to identify cases is inserted for comparison

\begin{tabular}{|c|c|c|c|c|c|}
\hline & $\begin{array}{l}\text { no of } \\
\text { cases }\end{array}$ & $\begin{array}{l}\text { Sensitivity (\%) } \\
\text { at } 80 \% \text { specificity }\end{array}$ & $\begin{array}{l}\text { Sensitivity (\%) } \\
\text { at } 90 \% \text { specificity }\end{array}$ & $A U C(95 \% C I)$ & $\begin{array}{l}\text { Best cut } \\
\text { off value }\end{array}$ \\
\hline \multicolumn{6}{|l|}{ All cases: } \\
\hline Clinicians' scores & 133 & 66 & 62 & $0.78(0.70-0.85)$ & 1 \\
\hline CDT & 150 & 43 & 35 & $0.67(0.59-0.75)$ & 5.4 \\
\hline GT & 158 & 26 & 25 & $0.57(0.49-0.65)$ & 44 \\
\hline ASAT & 157 & 40 & 30 & $0.67(0.59-0.74)$ & 26 \\
\hline ALAT & 157 & 25 & 21 & $0.63(0.55-0.70)$ & 28 \\
\hline ASAT/ALAT ratio & 148 & 17 & 4 & $0.54(0.45-0.62)$ & 0.79 \\
\hline \multicolumn{6}{|l|}{ Men: } \\
\hline Clinicians' scores & 75 & 72 & 69 & $0.84(0.73-0.91)$ & 0 \\
\hline CDT & 83 & 62 & 48 & $0.78(0.67-0.83)$ & 6.6 \\
\hline GT & 83 & 31 & 31 & $0.58(0.46-0.69)$ & 112 \\
\hline ASAT & 82 & 37 & 31 & $0.66(0.54-0.76)$ & 25 \\
\hline ALAT & 82 & 28 & 23 & $0.63(0.51-0.73)$ & \\
\hline ASAT/ALAT ratio & 82 & 17 & 9 & $0.54(0.46-0.63)$ & 0,76 \\
\hline \multicolumn{6}{|l|}{ Women: } \\
\hline Clinicians' scores & 59 & 55 & 50 & $0.71(0.58-0.82)$ & 1 \\
\hline CDT & 71 & 28 & 22 & $0.52(0.40-0.64)$ & \\
\hline GT & 75 & 17 & 6 & $0.51(0.39-0.63)$ & \\
\hline ASAT & 75 & 50 & 28 & $0.66(0.54-0.77)$ & 26 \\
\hline ALAT & 75 & 22 & 17 & $0.57(0.45-0.69)$ & 26 \\
\hline ASAT/ALAT ratio & 75 & 11 & 6 & $0.58(0.46-0.70)$ & 0.86 \\
\hline
\end{tabular}

AUC $=$ Area under the ROC curve. 
$\mathrm{p}=0.001)$ and GGT $\left(r_{\mathrm{s}}=0.12, \mathrm{NS}\right)$. However, the validity of this combination was poorer (LR 1.69) than that of \%CDT alone (LR 2.86). The agreement of clinicians' scores of a presumed alcohol relation with the combination was similar $(\kappa=0.25)$ to that of $\% \mathrm{CDT}$ alone $\kappa(=0.28)$.

CDT IN WITHDRAWAL SEIZURES

Alcohol withdrawal seizures were identified in a subgroup of 33 patients ( 23 men, 10 women, mean age 41 years). By definition, these patients were AUDIT positive and had a recent ( $<72$ hours) alcohol intake. As they all were AUDIT positive, specificity levels could not be calculated. Sensitivity of \%CDT in the withdrawal seizure subgroup was $52 \%$.

\section{CDT CONCENTRATIONS INFLUENCED BY AEDS}

Among 52 patients taking AEDs, accuracy of $\%$ CDT (sensitivity $30 \%$, specificity $76 \%$, LR 1.3) was poorer than for the 98 patients not taking AEDs (sensitivity $44 \%$, specificity $89 \%$, LR 4.0). \%CDT in the AED users (median 4.50) was not statistically different from $\% \mathrm{CDT}$ in those not taking AEDs (median 4.00, $\mathrm{p}=0.46)$. As patients with epilepsy consumed significantly less alcohol in the past week (mean 3.9 (SD 13.9) units) than others (19.3(3.75) units, $\mathrm{p}<0.0005)$, a difference could be expected. By comparison of the AUDIT negative groups we ensured low and comparable drinking levels in subgroups of AED users $(n=42)$ and patients not using AEDs ( $n=57)$. Between these groups, \%CDT was significantly higher among AED users (median 4.25) than the others (median 3.50, $\mathrm{p}=0.011)$. A significant difference $(\mathrm{p}=0.002)$ was also demonstrated between the smaller subgroups of abstainers (25 AED users, median 4.4 and 12 others, median 3.15). AUDIT negative patients were further subdivided by the enzyme inducing properties of the drugs. The 33 on enzyme inducing AEDs had significantly higher \%CDT (median 4.20) than the 57 patients not using AEDs (median 3.50, $p=0.014)$. AUDIT negative patients on AEDs not considered to be enzyme inducing $(n=9)$ did not differ from those who were not using AEDs $(p=0.23)$. Enzyme induction was documented by significantly higher GGT concentrations among the patients on enzyme inducing AEDs (median 80), than those not using AEDs (median 32, p<0.0005). GGT did not differ between the group using non-enzyme inducing drugs (median 31) and those not on AEDs $(p=0.32)$. Between AUDIT negative patients on enzyme inducing and non-enzyme inducing AEDs, no differences were found in mean alcohol intake in the past week $(p=0.21)$, or between proportions of abstainers $(p=0.78)$.

CAUSES OF FALSE POSITIVE CDT OR FALSE NEGATIVE AUDIT

Sixteen patients had \%CDT above the decision limit of $5.0 \%$ although being AUDIT negative. Among them were 10 patients taking AEDs $(56 \%)$ of whom six $(38 \%)$ were using enzyme inducing drugs and had increased GGT. AUDIT scores in these six patients were 0 or 1 and none of them reported any alcohol intake during the past week. Exclusion of these cases improved specificity of \%CDT from $86 \%$ to $90 \%$. Four out of the six false positive cases had $\%$ CDT values above $6.0 \%$. In one of the AED users, and six other patients, a close post hoc examination of hospital records, partly with information obtained from later admissions, suggested that faulty answers or underestimation of alcohol problems caused a negative AUDIT score. Out of the four cases that remained unexplained, three were taking AEDs not considered to be enzyme inducing and had GGT values within reference limits.

CAUSES OF FALSE NEGATIVE CDT OR FALSE POSITIVE AUDIT

In the 30 AUDIT positive patients who had $\%$ CDT values lower than $5.0 \%$, low recent alcohol consumption was probably the main cause. Five of them reported no consumption at all during the past week. Out of the remaining 25,17 consumed less than 18 units in the past week, equal to a mean daily intake below $40 \mathrm{~g}$ ethanol. Eight cases remained unexplained, as aggravation of alcohol problems leading to false positive AUDIT was not considered a realistic option.

\section{Discussion}

Our findings indicate that CDT is the best biochemical marker for alcohol related seizures. Higher specificity was found for s-ethanol and, unexpectedly, for ASAT, but at much lower sensitivity levels. However, in our unselected seizure population CDT detected only four out of 10 patients with a questionnaire based diagnosis of alcohol related seizures. These results are well in accordance with other studies on consecutive or mixed patient samples or patients with hazardous drinking levels. ${ }^{1011} 13$ 21-23 Thus, CDT seems to be of limited value in identifying hazardous drinking levels in an unselected medical population. The modest sensitivity levels, as found in our material and generally reported, should be recognised by the clinician as an important pitfall in the diagnostic investigation of any potentially alcohol related disease.

Although a markedly lower sensitivity of GGT than of CDT was found, the AUCs were not significantly different. However, AUC is a function of the performance of a marker at all possible sensitivity and specificity levels. In clinical practice, high specificity is preferred, as a false allegation of alcohol misuse is clearly unwanted. In a medicolegal context, a high specificity becomes particularly important. Thus, a more sensible comparison of markers would be sensitivity at predetermined high specificity levels. Such information can be derived from ROC curves, as shown in table 3. At $80 \%$ specificity, CDT outperforms GGT ( $43 \%$ and $27 \%$ sensitivity, respectively). Sensitivity of ASAT at $80 \%$ specificity was unexpectedly high, especially in women. Although well investigated in earlier studies, ASAT is not previously known to show such high sensitivity. Enzyme inducing drugs did not influence the sensitivity of ASAT. 
The two probably best markers of alcohol misuse, CDT and GGT, represent two metabolically unrelated responses to alcohol exposure. CDT probably identifies a different group of patients than GGT, as the two markers do not seem to be statistically associated. ${ }^{1022} 24$ Thus, combining the performances of the two markers to achieve higher accuracy is tempting. ${ }^{25}$ A recently proposed combination of CDT and $\mathrm{GGT}^{26}$ showed better validity than both CDT and GGT alone. The combination method used in that study ${ }^{27}$ has the advantage of providing a combined marker suitable for ROC analyses. Unfortunately, it is too complicated to be adopted into clinical practice. Under the assumption that advantages might be obtained simply by a clinical evaluation of CDT and GGT together, we investigated the performance of a simple combination of "positive \%CDT, and/or positive GGT". The increased sensitivity of this combination was obtained at the cost of reduced specificity, and its LR was poorer than that of \%CDT alone. Moreover, agreement of this combination with clinicians' assessment of alcohol's role in the aetiology of each seizure was poorer than that of \%CDT alone. Thus, our data do not allow recommendations as to the combined use of GGT and CDT.

Concomitant use of AEDs seems to increase CDT concentrations. We found that six out of 16 false positive CDT values might be attributable to this phenomenon. As six other patients probably had false negative AUDIT rather than false positive CDT, the influence of AEDs seemed to be the main cause of false positive CDT values in this material. To the best of our knowledge, this is the first report of CDT concentrations being influenced by concomitant medication. The present results allow little elaboration on the biochemical mechanisms causing increased CDT in response to antiepileptic medication. However, induction of liver enzymes seems to be one explanation. Previous studies of the effect of medication on CDT concentrations have shown negative results for hyperuricaemic agents, ${ }^{28}$ corticosteroids, $\beta$-blockers, ACE inhibitors, diuretics, $\mathrm{H} 2$-blocking agents, calcium antagonists, oral antidiabetics and insulin, and proton pump inhibitors. ${ }^{29}$ However, none of these drugs were enzyme inducing.

A range of hitherto poorly explored metabolic or endocrine associations of the various isoforms of transferrin have been proposed. ${ }^{9}{ }^{24}$ For instance, abstaining women have higher baseline CDT concentrations than men. ${ }^{80-32}$ There does not seem to be any simple association of sex hormones with CDT. Results differ as to the effects of both pregnancy ${ }^{33} 34$ and contraceptive pills. ${ }^{3132}$ Premenopausal women have higher CDT concentrations than postmenopausal women. ${ }^{24}{ }^{35}$ Subclinical iron deficiency, which is a common condition among women, may cause increased CDT concentrations, ${ }^{36}{ }^{37}$ as may iron reductive treatment. ${ }^{38}$ Increased transferrin concentrations and redirection of glycan synthesis to the highly sialylated transferrin forms seem to account for some of these differences. ${ }^{37}$ Insulin resistance is associated with high body mass index and has been postulated as one cause of reduced CDT sensitivity to alcohol intake. ${ }^{24} 39$ Finally, it should be kept in mind that ethanol, which obviously increases CDT concentrations, has enzyme inducing properties. If enzyme induction can be confirmed as an essential mechanism behind the influence of AEDs on CDT, then CDT may at least in part be increased by alcohol use through a similar mechanism.

The quantity, frequency, and duration of the alcohol consumption needed to cause increased CDT has not been well established. According to the manufacturer, daily alcohol consumption exceeding $60 \mathrm{~g}$ for periods longer than 2-4 weeks may result in \%CDT above $6.0 \%$. However, volunteers consuming this amount of alcohol or more for up to 1 month only produced increased CDT concentrations in a few of the subjects. ${ }^{40}$ These and other reports suggest that longer term alcohol misuse is needed to produce increased CDT. Patient recollection of exact alcohol consumption over such long time spans is unreliable, but similar information is easily and more reliably obtained by the AUDIT questionnaire. In this material, AUDIT data correlated equally well with \%CDT as the actual intake in the past week did (both correlations, $\mathrm{p}<0.0005$ ). Clinicians may have a lower threshold for performing a CDT test than for obtaining a thorough history of alcohol consumption, or using a structured questionnaire such as AUDIT. Our results indicate that such a policy should be discouraged, as CDT used as a stand alone marker may identify only half of the patients disclosed by AUDIT.

Early diagnosis and intervention is essential to stop processes that might cause irreversible damage to the nervous system. One study of patients with various neurological diseases indicated that CDT might be a valuable tool in the diagnostic investigation. ${ }^{12}$ However, disease groups were not studied separately, and to our knowledge, this is the first report in which CDT has been studied in patients with seizure.

There is no consensus as to the definition of an alcohol related seizure. The literature in this field suffers from the lack of a unanimous definition, ${ }^{41}{ }^{42}$ which makes the reproducibility of results difficult. Whereas the withdrawal seizure is closely linked to cessation or abrupt reduction of an increased alcohol intake, ${ }^{14}$ other alcohol related seizures may not be directly related to a drinking bout. ${ }^{5}$ We propose a simple and reproducible operational definition of alcohol related seizures as seizures occurring in subjects with hazardous alcohol consumption, as identified by a score of 8 or more on the AUDIT questionnaire. This questionnaire was designed to detect drinking levels at an early stage, before alcoholism is evident, and is, in this respect, more sensitive than other established questionnaires, such as $\mathrm{CAGE}^{43}$ and the Michigan alcoholism screening test (MAST). ${ }^{44}$

As the half life of CDT is about $12-17$ days $^{8} 9$ and most alcohol related seizures occur within 48 hours after the cessation of alcohol 
drinking, ${ }^{14}$ a significant decline in CDT at the time of admission to hospital is unlikely. In view of this, alcohol related seizures, and withdrawal seizures in particular, provide an ideal model for evaluation of the usefulness of CDT in the detection of neurological disease related to current or recent alcohol misuse. The subgroup of alcohol related seizure patients who fulfilled our criteria for withdrawal seizures, had a markedly higher mean intake during the past week. As expected, sensitivity of CDT was better for this group (52\%). However, an even higher sensitivity would be anticipated in this highly selected subgroup. An explanation for the modest sensitivity even for this group may be that our material contains few heavily misusing people. As even weekend binges can trigger seizures, ${ }^{4}$ the clinical diagnosis of a withdrawal seizure does not necessarily imply long term alcohol misuse. But, to be of help to clinicians, CDT should be more accurate when applied to this particular subgroup.

\section{Conclusion}

Our results show that although CDT seems to be the best performing trait marker for alcohol related seizures, sensitivity levels in a mixed seizure population are moderate. CDT only recognised $41 \%$ of patients diagnosed as having a hazardous drinking level. Even in withdrawal seizures, the sensitivity level of CDT was only $52 \%$. The treating clinician's overall ability to identify seizures related to alcohol use by routine investigations is better than that of any biomarker, but many cases are missed. Conversely, an even higher number of cases would be missed if the clinician should rely on CDT alone. However, the high specificity of an increased CDT clearly indicates that this index may be a useful supplement to a thorough clinical evaluation of seizures with obscure aetiology. Enzyme inducing AEDs were associated with increased CDT values and may be responsible for false positive cases. This apparent medication effect needs to be tested in designed studies.

The Norwegian Research Council supported the study, project No 111342/330. Axis Biochemicals ASA provided \%CDT-TIA kits for this study, and gave financial support for the purchase of CDTect kits as well as supporting the laboratory work. Roch Diagnostics contributed financially to the laboratory work. We thank our colleagues at the Department of Neurology, Trondheim University Hospital, for their kind cooperation in the study.

1 Bowman K, Jellinek E. Alcoholic mental disorders. In Jellinek E, ed. Alcohol addiction and chronic alcoholism. New Jellinek E, ed. Alcohol addiction and chronic alco

2 Lennox W. Alcohol and epilepsy. Quarterly fournal of Studies on Alcohol 1941;2:1-11.

3 Earnest M, Yarnell P. Seizure admissions to a city hospital: the role of alcohol. Epilepsia 1976;17:387-93.

4 Hillbom M. Occurrence of cerebral seizures provoked by alcohol abuse. Epilepsia 1980;21:459-66.

5 Bråthen G, Brodtkorb E, Helde G, et al. The diversity of seizures related to alcohol use. A study of consecutive patients. Eur F Neurol (in press). 1999;6:697-703.

6 Pieninkeroinen IP, Telakivi TM, Hillbom ME. Outcome in subjects with alcohol-provoked seizures. Alcohol Clin Exp Res 1992;16:955-9.

7 Hillbom M, Hjelm-Jaeger M. Should alcohol withdrawal seizures be treated with antiepileptic drugs? Acta Neurol Scand 1984;69:39-42.

8 Stibler H. Carbohydrate-deficient transferrin in serum: a new marker of potentially harmful alcohol consumption reviewed. Clin Chem 1991;37:2029-37.
9 Allen JP, Litten RZ, Anton RF, Cross GM. Carbohydratedeficient transferrin as a measure of immoderate drinking: remaining issues. Alcohol Clin Exp Res 1994;18: $799-812$.

10 Nilssen O, Huseby NE, Høyer G, et al. New alcohol markers: how useful are they in population studies: the Svalbard study 1988-9. Alcohol Clin Exp Res 1992;16: $82-6$.

11 Nyström M, Peräsalo J, Salaspuro M. Carbohydratedeficient transferrin (CDT) in serum as a possible ndicator of heavy drinking in young university students. Alcohol Clin Exp Res 1992;16:93-7.

12 Stibler H. Diagnosis of alcohol-related neurological diseases by analysis of carbohydrate-deficient transferrin in serum. Acta Neurol Scand 1993;88:279-83.

13 Sillanaukee P, Seppä K, Lof K, et al. CDT by anionexchange chromatography followed by RIA as a marker of heavy drinking among men. Alcohol Clin Exp Res 1993;17: 230-3.

14 Victor M, Brausch C. The role of abstinence in the genesis of alcoholic epilepsy. Epilepsia 1967;8:1-20.

$15 \mathrm{Ng} \mathrm{S}$, Hauser W, Brust J, et al. Alcohol consumption and withdrawal in new-onset seizures. $N$ Engl F Med 1988;319: 666-73.

16 Saunders JB, Aasland OG, Babor TF, et al. Development of the alcohol use disorders identification test (AUDIT): WHO collaborative project on early detection of persons with harmful alcohol consumption: II. Addiction 1993;88: 791-804.

17 Conigrave K, Hall W, Saunders J. The AUDIT questionnaire: choosing a cut-off score. Addiction 1995;90: 1349-56

18 MacKenzie D, Langa A, Brown T. Identifying hazardous or harmful alcohol use in medical admissions: a comparison of AUDIT, CAGE, and BRIEF MAST. Alcohol Alcohol 1996;31:591-9.

19 Turner C. How much is a "standard drink"? An analysis of 125 studies. British fournal of Addiction 1990;85:1171-5.

20 Gram L. Pharmacokinetics of new antiepileptic drugs. Epilepsia 1996;37(suppl 6):S12-16.

21 Bell H, Tallaksen CM, Try K, et al. Carbohydrate-deficient transferrin and other markers of high alcohol consumption: a study of 502 patients admitted consecutively to a medical department. Alcohol Clin Exp Res 1994;18:1103-8.

22 Löf K, Seppä K, Itälä L, et al. Carbohydrate-deficient transferrin as an alcohol marker among female heavy drinkers: a population-based study. Alcohol Clin Exp Res 1994;18:88994.

23 Godsell P, Whitfield J, Conigrave K, et al. Carbohydrate deficient transferrin levels in hazardous alcohol consumption. Alcohol Alcohol 1995;30:61-6.

24 Whitfield J, Fletcher L, Murphy T, et al. Smoking, obesity, and hypertension alter the dose-response curve and test sensitivity of carbohydrate-deficient transferrin as a marker of alcohol intake. Clin Chem 1998;44:2480-9.

25 Helander A, Carlsson AV, Borg S. Longitudinal comparison of carbohydrate-deficient transferrin and $\gamma$-glutamyl transferase: complementary markers of excessive alcohol consumption. Alcohol Alcohol 1996;31:101-7.

26 Huseby N, Nilssen O, Kanitz R-D. Evaluation of two biological markers combined as a parameter of alcohol dependency. Alcohol Alcohol 1997;32:731-7.

27 Shultz E. Multivariate receiver-operating characteristic curve analysis: prostate cancer screening as an example. Clin Chem 1995;41:1248-55.

28 Kaneko K, Fujimori S, Yamanaka H, et al. Effect of hypouricemic agents on serum carbohydrate-deficient transferrin in gouty patients. Adv Exp Med Biol 1994;370:27-30.

29 Meerkerk G-J, Njoo KH, Bongers IMB, et al. The specificity of the CDT assay in general practice: the nfluence of common chronic diseases and medication on the serum CDT concentration. Alcohol Clin Exp Res 1998; 22:908-13.

30 Anton RF, Moak DH. Carbohydrate-deficient transferrin and $\gamma$-glutamyltransferase as markers of heavy alcohol consumption: gender differences. Alcohol Clin Exp Res 1994;18:747-54.

31 Bell H, Tallaksen C, Sjåheim T, et al. Serum carbohydratedeficient transferrin as a marker of alcohol consumption in patients with chronic liver diseases. Alcohol Clin Exp Res 1993;17:246-52.

32 Helander A, Vabö E, Levin K, et al. Intra- and interindividual variability of carbohydrate-deficient transferrin, $\gamma$-glutamyltransferase, and mean corpuscular volume in $\gamma$-glutamyltransferase, and mean corpuscular

33 Stibler H, Borg S, Joustra M. Micro anion exchange chromatography of carbohydrate-deficient transferrin in serum in relation to alcohol consumption (Swedish Patent 8400587-5). Alcohol Clin Exp Res 1986;10:535-44.

34 Stauber RE, Jauk B, Fickert P, et al. Increased carbohydratedeficient transferrin during pregnancy: relation to sex hormones. Alcohol Alcohol 1996;31:389-92.

35 LaGrange L, Anton RF, Garcia S, et al. Carbohydratedeficient transferrin levels in a female population. Alcohol Clin Exp Res 1995;19:100-3.

36 Stauber RE, Vollmann H, Pesserl I, et al. Carbohydratedeficient transferrin in healthy women: relation to estrogens and iron status. Alcohol Clin Exp Res 1996;20: 1114-7.

37 deJong G, van Noort W, Feelders R, et al. Adaptation of transferrin proteins and glycan synthesis. Clin Chim Acta 1992;212:27-45. 
38 Jensen PD, Peterslund NA, Poulsen JH, et al. The effect of iron overload and iron reductive treatment on the serum concentration of carbohy

39 Fagerberg B, Agewall S, Urbanavicius V, et al. Carbohydrate-deficient transferrin is associated with insulin sensitivity in hypertensive men. $\mathcal{F}$ Clin Endocrinol Metab 1994;79:712-5

40 Lesch OM, Walter H, Antal J, et al. Carbohydrate-deficient transferrin as a marker of alcohol intake: a study with healthy subjects. Alcohol Alcohol 1996;31:265-71.
41 Chan A. Alcoholism and epilepsy. Epilepsia 1985;26:323-33. 42 Devetag F, Mandich G, Zaiotti G, et al. Alcoholic epilepsy: of a ries and proposed classification and etiopathogenesis. Ital f Neurol Sci 1983;3:275-84.

3 Mayfield D, McLeod G, Hall P. The CAGE questionnaire: validation of a new alcoholism screening instrument. $A m \mathcal{F}$ Psychiatry 1974;131:1121-3.

44 Yersin B, Trisconi Y, Paccaud F, et al. Accuracy of the Michigan alcoholism screening test for screening of alcoholism in patients of a medical department. Arch Intern Med 1989;149:2071-4.

\section{HISTORICAL NOTE}

\section{Alzheimer's disease}

At a psychiatric hospital in Frankfurt, a 51 year old woman (Auguste D), sought help because her family had noticed that she had become uncharacteristically jealous of her husband, and soon became forgetful and tended to get lost. She was fortunate to end up in the hands of Alois Alzheimer, in November $1901 .{ }^{1}$ When she eventually died in 1906, her brain was sent to Alzheimer, then working in Munich. Alzheimer reported the necropsy of 8 April 1906 to a conference in Tübingen, on 3 and 4 November 1906. An abstract of this report was published in 1907 : The Frankfurt archive* has recently been recovered.

"A woman, 51 years old, showed jealousy towards her husband as the first noticeable sign of the disease. Soon a rapidly increasing loss of memory could be noticed. She could not find her way around in her own apartment. She carried objects back and forth and hid them. At times she would think that someone wanted to kill her and would begin shrieking loudly. In the institution her behaviour bore the stamp of utter perplexity. . . . Periodically she was totally delirious, dragged her bedding around, called her husband and her daughter, and seemed to have auditory hallucinations.

.. "The generalised dementia progressed however. . . . After 4 years of the disease death occurred. At the end, the patient was completely stuporose; she lay in her bed with her legs drawn up under her ...' The necropsy disclosed a generally atrophic brain without macroscopic lesions. The large brain vessels were altered by arteriosclerosis. Alzheimer found

“. . .peculiar changes of the neurofibrils...(which) are eventually seen clustering together in thick bundles which emerge at the surface of the cell and miliary foci distinguishable by the deposit in the cerebral cortex of a peculiar substance which can be recognised without stain and is, in fact, very refractory to staining." 3

Later these changes were called respectively neurofibrillary tangles and amyloid plaques. Alzheimer did not claim that he had found a new disease. ${ }^{4} \mathrm{He}$ was impressed by the histological variation of the neuropathological process, which he did not yet understand. He was keen to show the value of newer histological techniques in psychiatric illnesses that he believed resulted from organic brain diseases.

A year later, his colleague Francesco Bonfiglio (1883-1966) published a second case, and two years later another Italian, Gaetano Perusini (1879-1915), described four cases: two of them were the cases described by Alzheimer and Bonfiglio. They too believed that they had observed an unusual variant of senile dementia. Emil Kraepelin introduced the term Alzheimer's disease, in the 8 th edition of his textbook Compendium der Psychiatrie (1910). Kraepelin knew of Alzheimer's two cases, but also knew of Gaetano Perusini's two new cases. Kraepelin thought that these four patients were significantly different from the usual senile dementias; therefore he categorised them as presenile dementia. Alzheimer did not share this view at this stage. A case he described in 1911 showed no neurofibrillary tangles, and he thought that the plaques were less important. He had found that plaques and tangles were sometimes inconspicuous in patients with severe dementia. Thus began a lasting argument: Was Alzheimer's disease the same as, or was it a variant of senile dementia? Was the disease he had found simply an aging process, or was there some other degenerative factor in operation? ${ }^{5}$

Alzheimer's disease now covers a range stretching from the presenile into the senile period. The $\beta$-amyloid peptides at the core of senile plaques come from an amyloid precursor protein APP that plays a part in neuronal growth and neurotoxicity. ${ }^{6}$ Chromosome 21 , the site of the trisomy of Down's syndrome, contains the gene for APP. Mutations in presenilin (PS)-1 and -2, located on chromosome 14 and 1 respectively, are the major association with early onset familial Alzheimer's disease (FAD). FAD has also been linked to mutations in the amyloid $\beta$ precursor protein $(\beta \mathrm{PP})$. The presence of the apolipoprotein E4 allele on chromosome 19 is a risk factor for late onset $\mathrm{AD} .^{7}$ There is evidence of an $\mathrm{AD}$ susceptibility locus on chromosome $12^{8}$ and possibly on other chromosomes.

Alzheimer was born at Ochsenfurter Strasse $15 \mathrm{~A}$ in Marktbreit-am-Main, the son of a notary. He attended medical schools in Berlin, Wurzburg, and Tubingen, becoming an internist at Frankfurt in 1887-8. He was joined by Nissl, starting a lifetime collaboration. Emil Kraepelin (1856-1926), known as the Linnaeus of Psychiatry, expressed scepticism about pathology to Oskar Vogt: "anatomy can contribute nothing to psychiatry." Despite this, Kraepelin attracted first Nissl, then young Alzheimer to Heidelberg in 1902. Alzheimer devoted his attention to the histopathology of general paralysis for the insane (GPI), dementia, and cerebral arteriosclerosis.

He worked with Wilhelm Erb (1840-1920) and became his close friend. In 1894, Alzheimer received a telegram from Erb, who accompanied Otto Geisenheimer, a banking director, on a scientific expedition in Africa. Geisenheimer suffered a crisis of GPI, in which Alzheimer had become a recognised expert. Alzheimer hurried to Algeria, but his efforts were in vain. However, Geisenheimer's widow, Cecile, weeks after her husband's death, asked Alzheimer to marry her. They had three children but she predeceased him.

Alzheimer followed Kraepelin to Munich in the Anatomisches Laboratorium. Popular as a teacher, he spent hours poring down the microscope, always a cigar, forgotten and unfinished on the bench. As he moved to the next student another cigar was lit and quickly placed at his side; by the end of the day 20 or more large cigar stumps were strewn around the laboratory.

He was instrumental in the late conversion of Kraepelin to clinical-anatomical correlation in psychiatry. He moved to the chair of psychiatry in Breslau in 1912, but on arrival was taken to hospital with cardiac symptoms that delayed the start of his work. During the first world war he was without assistants and gradually longstanding rheumatic carditis relapsed; and he died in 1915, aged 52.

Additional biography:

Hoff P. Alois Alzheimer 1864-1915. An overview of his life and work on the occasion of his 125th birthday. Nervenarzt 1989;60:332-7. Kircher T, Wormstall H. Alois Alzheimer (1864-1915): student days and first scientific activities. F Geriatr Psychiatry Neurol 1997;10:127-9

J M S PEARCE

304 Beverly Road, Alunby, Hull HU10 7BG, UK

1 Graeber MB. No man alone: the rediscovery of Alois Alzheimer's original cases. Brain Pathol 1999;9:237-40

2 Maurer K, Volk S, Gerbaldo H. Auguste D and Alzheimer's disease. Lancet 1997;349:1546-9.

3 Alzheimer A. Ueber eine eigenartige Erkrankung der Hirnrinde. Allgemeine Zeitschrift für Psychiatrie Neurol 1907;64:146-8.

4 Alzheimer A. Alzheimer's disease: presenile dementia. Preliminary note in Neurologie Zentralblatt 1906;25:1134. Translated in: Arch Neurol 1969;21:109-10.

5 Arts NJM. Alzheimer's disease In: Koehler PJ, Bruyn GW, Pearce JMS, eds. Common eponyms in neurological practice. Oxford: Oxford University Press, 2000 (in press).

6 Sheng JG, Zhou XQ, Mrak RE, et al. Progressive neuronal injury associated with amyloid plaque formation in Alzheimer disease. $7 \mathrm{Neu}$ ropathol Exp Neurol 1998;57:714-7.

7 Wisniewski T, Dowjat WK, Permanne B, et al. Presenilin-1 is associated with Alzheimer's disease amyloid. Am f Pathol 1997;151:601-10.

8 Rogaeva E, Premkumar S, Song Y, et al. Evidence for an Alzheimer disease susceptibility locus on chromosome 12 and for further locus heterogeneity. $\mathcal{F A M A}$ 1998;280:614-8.

9 Haymaker W, Schiller F, eds. The founders of neurology, 2nd ed. Springfield. Charles C Thomas, 1970:315-8.

*In 1992 and 1997, the histological slides of Alzheimer's original cases were rediscovered and re-examined in Munich. This material was found at the Institute of Nurop thound at the Institute of Neuropathology, Munich. Kohshiro Eujisawa of Tokyo had through Henry deE Webster of the NIH in Bethesda, prompted the rediscovery of Alzheimer's cases. Histological and molecular genetic findings obtained on the tissue sections have been reported (Neurogenetics 1997;1:73-80;
1998;1:223-28). 